\section{Top tips for your feline eye examination}

\section{David Maggs}

Ready visualisation of almost all parts of the eye means nothing can replace a complete examination. Fortunately, a thorough ophthalmic examination is readily performed with just four guidelines, four skills and inexpensive equipment.

The four essential requirements:

- The patient and examiner must be at eye level with each other (plus lower the cat's head to permit examination of the ventral globe and anterior chamber)

- The examination must be performed in dim ambient light

- A bright, focal light source (e.g. Finoff ${ }^{\circledR}$ transilluminator) and a means of magnification (e.g. Optivisor ${ }^{\circledR}$ head loupe) are essential

- Always perform an orderly and complete examination. (Examine the unaffected eye prior to the affected eye and use a prepared examination sheet)

The four essential skills:

- Retroillumination (excellent for assessing pupil symmetry and clarity of the clear ocular media/ structures)

- Focal illumination or transillumination (excellent for depth perception)

- Tonometry - measurement of intraocular pressure (IOP), and essential for diagnosing uveitis (typically low IOP), glaucoma (always high IOP) and conjunctivitis (always normal IOP)

- Assessment of aqueous flare (pathognomonic for anterior uveitis)

Although visual examination of the eyes frequently provides all of the clues necessary to reach a clinical diagnosis, the Schirmer tear test (STT), fluorescein staining, globe retropulsion and cranial nerve testing are also routine

The Schirmer tear test (STT) strip should be placed in the lateral, ventral conjunctival fornix for 1 minute so that the strip lightly abrades the cornea and produces reflex tearing. The STT must be performed prior to application of any topical solutions. The median value for cats is $18 \mathrm{~mm} / \mathrm{min}$ but there is wide variation among cats (normal range 9-34 mm/ min) but also between tests for the same cat.

Ulcer diagnosis is best achieved if a very small amount of fluorescein dye is applied. A few drops of irrigation solution are trickled over a fluorescein-impregnated strip; the strip is then dripped/blotted dry and very lightly touched onto the bulbar conjunctiva. The eye is then examined with cobalt blue light. Flushing is not necessary.

Testing of cranial nerves involved in ocular function (CN II-VII) can be performed with:

Menace response (CN II and VII, visual cortex and cerebellum)
- Behavioural vision testing (CN II, visual cortex and all areas involved in motor function)

- Direct and consensual pupillary light response (PLR) (CN II \& III and central visual pathways excluding the visual cortex)

- Palpebral and corneal reflexes (CN V and VII)

- Dazzle reflex (CN II and VII, and subcortical visual pathways)

- Doll's eye reflex (CN III, IV and VI)

Retropulsion of the globe is essential for investigating orbital disease. Gentle digital pressure is applied to both globes in a variety of directions through closed lids. The resistance to retropulsion and the resilience with which the globes 'spring' back against the retropulsive force are subjectively assessed.

Every red eye (conjunctivitis vs. keratitis vs. uveitis vs. glaucoma) should undergo:

Retroillumination (assess for anisocoria)
PLR testing
STT
Flare assessment
Tonometry
Fluorescein staining

\section{KEY LEARNING OBJECTIVES}

- To apply the four essential requirements of a thorough ophthalmic examination

- To apply the four essential skills for a thorough ophthalmic examination

- To list at least four tests that should be performed on a reddened eye

\section{MULTIPLE CHOICE OUESTIONS}

1. What should an eye examination include?
a. PCR testing for FHV-1, fluorescein staining, Schirmer tear testing and retroillumination
b. Tonometry, retroillumination, fluorescein staining and conjunctival culture
c. Retroillumination, transillumination, assessment of aqueous flare and tonometry
d. Nasolacrimal duct flushing, fluorescein staining, globe retropulsion and cranial nerve testing

2. What are the requirements for a basic eye

examination?
a. Include that the cat should be placed at eye level with the examiner
b. Are different for a dog and a cat
c. Include a well lit room
d. Are expensive and not easily met in a general practice

3. Which of the following is true for reddened eyes in cats?
a. Do not need their intraocular pressures taken
b. Should be examined for pupil asymmetry
c. Represent an exception to the rule that the ophthalmic examination should generally be conducted in a darkened room

d. Should have their nasolacrimal duct flushed 
4. Which of the following is true for the Schirmer tear test in cats?

a. Is difficult to interpret due to poor repeatability and wide normal variability

b. Should be performed after application of a topical anaesthetic

c. Should be performed in the ventromedial conjunctival fornix d. Has a median (and range) in normal cats of $5(0-16) \mathrm{mm} / \mathrm{min}$

5. Which of the following is true for fluorescein staining?

a. Should be performed before the STT

b. Is of no value in eyes with normal IOP

c. Is necessary only when corneal changes are noted during the examination

d. If correctly performed does not need flushing

\section{FHV-1 and cat corneas: are you up to date?}

\section{David Maggs}

Feline herpesvirus (FHV-1) is a ubiquitous virus with relatively consistent virulence worldwide and, yet, we see a huge range of clinical signs in infected cats. The most likely reason for this is the variable host response to this virus. Naiive kittens infected against a backdrop of waning maternal immunity almost inevitably get severe upper respiratory and bilateral ocular disease with high morbidity but rare mortality. By contrast, adult cats can undergo viral reactivation with viral shedding that can infect in-contact cats but without demonstrating clinical signs themselves, or can develop debilitating but often unilateral ocular, respiratory or dermatological syndromes. These scenarios demonstrate the broad diversity of clinical signs seen with $\mathrm{FHV}-1$ in clinical practice. An understanding that normal cats frequently shed virus also explains why virological testing is of little diagnostic value.

Although FHV-1 preferentially infects conjunctival cells, corneal disease (either through direct herpetic infection or as a consequence of marked conjunctivitis) is particularly important because it is painful, often not completely reversible and potentially blinding. The pathognomonic sign of corneal herpetic disease is dendritic ulcers; however these are often missed as they are transient and occur early following infection or reactivation before rapidly coalescing to form geographic ulcers. If conjunctival ulceration occurs concurrently, symblepharon formation is possible. Although recrudescent disease occurs in a minority of latently infected cats, it produces serious corneal stromal and epithelial diseases which may be immunopathological (i.e. immune-mediated, but not necessarily autoimmune - such as herpetic stromal keratitis or eosinophilic keratitis), cytolytic (i.e. ulcerative) or metaherpetic (i.e. resulting from structural tissue damage subsequent to cytolytic or immunopathological disease). The concept of metaherpetic disease is relatively new in veterinary medicine and is exemplified by tear film deficiency secondary to herpetic damage of lacrimal nerves or ablation of conjunctival goblet cells responsible for tear mucins. Determining which of these three pathogenic mechanisms is likely is critical for selection of effective therapies.

Therapy for herpetic disease can be supportive (such as antibiotics for treatment or prophylaxis of secondary infection, tear substitutes for dry eye, lysine as an adjunctive therapy that may limit viral replication, or the interferons which alter the immune state to be more resistant to viruses) or topically or systemically administered antiviral agents (such as famciclovir, cidofovir, ganciclovir, idoxuridine, trifluridine or aciclovir). The following are some important updates about these therapies:

Lysine should be given twice daily (500 mg orally) and NOT applied to food such that cats 'graze' it throughout the day

- There is minimal peer-reviewed evidence to support systemic or topical use of the interferons

- Famciclovir - a prodrug of penciclovir - is one of the best studied antiviral agents in cats, and when given at $90 \mathrm{mg} / \mathrm{kg}$ orally q12h creates effective tear penciclovir concentrations. Do not taper dose!

- All antiviral drugs are virostatic; therefore they are ineffective against latent virus and must be given very frequently to be effective

- FHV-1 causes long-term ablation of the conjunctival goblet cells that is not prevented or corrected with famciclovir. Topical hyaluronate may aid regeneration of conjunctival goblet cells and is an essential adjunctive therapy in cats with herpetic disease

\section{KEY LEARNING OBJECTIVES}

- To apply some basic virology in the diagnosis and treatment of FHV-1 in cats

- To understand the limitations of virostatic drugs and how these are minimised by therapeutic choices

- To understand the importance of tear film dysfunction and augmentation in feline herpetic disease

\section{MULTIPLE CHOICE QUESTIONS}

1. Which of the following statements about feline herpesvirus infection in cats is true?

a. Rarely establishes latency

b. Recurs throughout life with similar bilateral signs to those seen at first infection

c. Typically causes severe bilateral signs in cats infected for the first time

d. Is typically recurrent only during the first 2-3 years after primary infection

2. Which of the following statements about feline herpesvirus infection in cats is true?

a. Always produces ulcerative corneal disease

b. Has been associated with feline eosinophilic keratitis

c. Is not associated with corneal sequestrum development

d. Should be treated topically 